\title{
Mikrobielle Gemeinschaften
}

\section{Bakterielle Multizellularität in Biofilmen}

\section{KNUT DRESCHER}

MAX-PLANCK-INSTITUT FÜR TERRESTRISCHE MIKROBIOLOGIE, MARBURG; FACHBEREICH PHYSIK, UNIVERSITÄT MARBURG

\section{VAAM-Forschungspreis 2019}

\section{Bacteria have been extensively investigated in their unicellular mode of life in liquid cultures. However, biofilm communities are now considered to be the most abundant mode of bacterial life on Earth, and many pro- perties and functions of biofilms are still unclear. Mechanistically under- standing which properties and functions of biofilms emerge at the single- cell level, and which emerge at the multicellular level, presents a major challenge for biofilm research in the coming years.}

DOI: $10.1007 / \mathrm{s} 12268-019-1038-6$

(C) Der Autor 2019

Grundlegende Erkenntnisse über das Leben von Bakterien und deren Evolution auf unserer Erde beziehen sich bislang weitestgehend auf ihre einzellige Lebensweise. Die Entdeckung der bakteriellen Kommunikation (Quorum sensing) Anfang der 1970er-Jahre [1] veränderte unsere Blickweise auf Bakterien grundsätzlich: Mithilfe von Botenstoffen können Bakterien nicht nur mit Artgenossen, sondern auch mit anderen Spezies interagieren, um gemeinschaftliches Verhalten wie Biolumineszenz oder Virulenz zu koordinieren. Neben dem Quorum sensing können klonale bakterielle Populationen auch grundlegende Formen von Arbeitsteilung und Altruismus ausbilden [2] sowie sich räumlich in dynamischen Schwärmen oder Oberflächenangehefteten Biofilmen organisieren [3, 4]. Bakterielles Sozialverhalten ist nicht beschränkt auf wenige Spezies, sondern weitverbreitet. Die Vielfalt des möglichen Sozialverhaltens und der Wechselwirkungen zwischen Bakterien ist in Multi-Spezies-Gemeinschaften besonders groß [2].

\section{Mikrobielle Biofilme}

Insbesondere die Biofilmbildung stellte sich in den letzten Jahren als bakterielles Sozialverhalten von enormer Bedeutung heraus. Belastbare Messungen der Zusammensetzung und Besiedlungsdichte vieler repräsentativer Ökosysteme zeigten, dass die meisten Mikroorganismen auf unserem Planeten in Biofilmen leben [5]. Biofilme machen einen Großteil der Biomasse in terrestrischen sowie marinen Ökosystemen aus und leisten somit einen erheblichen Beitrag zu den biogeochemischen Zyklen der Erde [4]. Sie haben auch eine groBe medizinische Bedeutung, da viele chronische und einige akute Infektionen sowie Implantat-assoziierte Infektionen und Wundinfektionen auf Biofilme zurückzuführen sind [6]. Zudem beeinflussen Biofilme die Gesundheit indirekt. Sie treten beispielsweise in Sanitäranlagen als schleimige Beläge auf, sind durch Desinfektionsmittel nur schwer zu entfernen und begünstigen somit die Übertragung von Keimen in Wohnungen und Kliniken [6].

Biofilme sind Oberflächen-assoziierte mikrobielle Gemeinschaften, in denen Zellen durch eine extrazelluläre Matrix miteinander verbunden sind. Bestandteile der Matrix können Proteine, Polysaccharide, Nukleinsäuren oder auch Lipide sein [4]. Die genaue Zusammensetzung der Matrix variiert stark in verschiedenen Spezies, wodurch die mechanischen Eigenschaften von Biofilmen sehr unterschiedlich sein können, von eher viskoelastischen, schleimigen Biofilmen bis zu festen oder brüchigen. Basierend auf den
Pionierarbeiten von Bill Costerton in den späten 1970er-Jahren wurden Gemeinsamkeiten im Prozess der Biofilmbildung von verschiedenen Spezies identifiziert: Zunächst heften sich Bakterien reversibel an eine Oberfläche an. Dies geht in eine irreversible Adhäsion über, gefolgt von der Produktion einer extrazellulären Matrix und dreidimensionalem Wachstum der Zellgemeinschaft.

Als Konsequenz des dreidimensionalen Wachstums entstehen räumliche Gradienten vieler Stoffe, abhängig von der Größe der Gemeinschaft. Zellen am Rand des Biofilms haben deutlich besseren Zugang zu Nährstoffen als Zellen im Inneren des Biofilms $[2,4]$, zum einen wegen der extrazellulären Matrix, welche die Diffusion in den Biofilm erschwert, zum anderen wegen des Verbrauchs von Nährstoffen in den Zellen der äußeren Bereiche der Gemeinschaft. Daneben kann die Konzentration von Abfallprodukten des Stoffwechsels sowie die Verfügbarkeit von Botenstoffen innerhalb der Gemeinschaften räumlich variieren. Diese Vielzahl von Gradienten führt dazu, dass selbst klonale Populationen räumlich strukturiert phänotypisch heterogen sein können. Die Auswirkungen dieser räumlichen Gradienten auf das Verhalten von einzelnen Zellen in Biofilmen und der Biofilmgemeinschaft als Ganzes sind bisher kaum verstanden.

\section{Sind Biofilme eine Ansammlung} von Einzellern oder mehr?

Wenn eine bakterielle Gemeinschaft aus mehreren räumlich strukturierten Zelltypen besteht, von denen sich jeder womöglich in einer anderen mikroskopischen Umwelt befindet, ist es nicht einfach, vorherzusagen, wie sich diese Gemeinschaft verhält: Ist die Stressantwort eines Biofilms die Summe der Stressantworten aller einzelnen Zellen im Biofilm, ähnlich zum Verhalten von Bakterien in geschüttelter Flüssigkultur? Oder entwickeln sich neue Verhaltensweisen von Zellen in Biofilmen, sodass die Stressantwort eines Biofilms nicht der Summe der individuellen Stressantworten entspricht? 

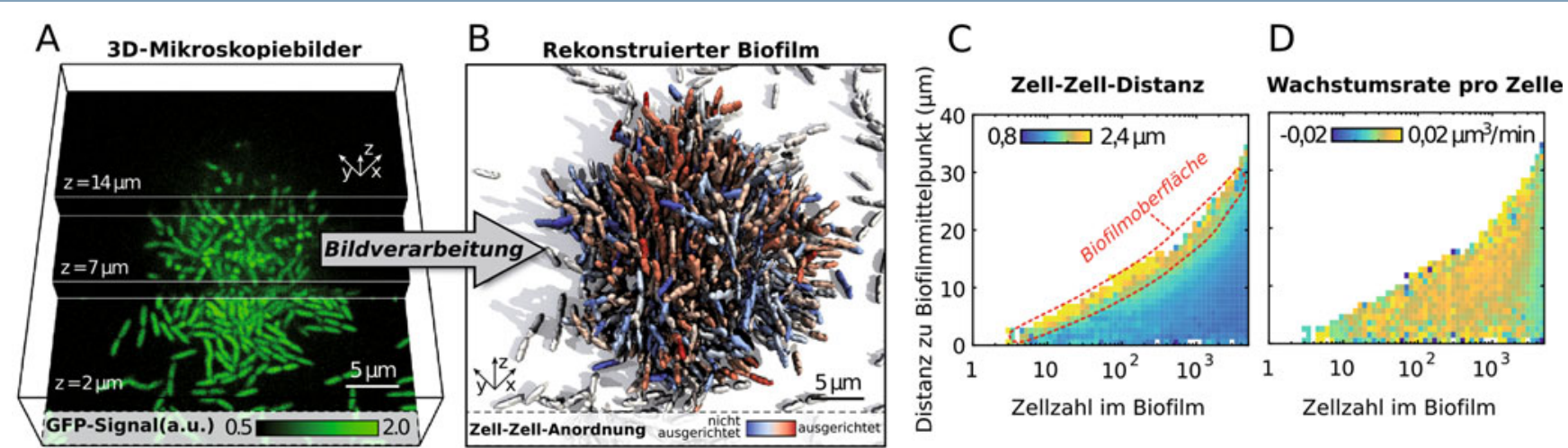

$\Delta$ Abb. 1: Quantifizierung von Biofilmeigenschaften auf Einzelzellebene. A, dreidimensionale Konfokalmikroskopiebilder von einem Vibrio choleraeBiofilm. B, Automatisierte Bildverarbeitung ermöglicht Segmentierung aller einzelnen Zellen. Quantifizierung der Zell-Zell-Distanz (C) und der zellulären Wachstumsrate (D) in Abhängigkeit von der Zellzahl im Biofilm und der Distanz von jeder Zelle zum Biofilmmittelpunkt auf der Substratebene (modifiziert nach [9]).

Es gibt Beispiele dafür, dass sich Zellgemeinschaften in Biofilmen anders verhalten als eine vergleichbar große Anzahl einzelner Zellen. Ein wichtiges Beispiel dafür ist die Toleranz von Biofilmen gegenüber Antibiotika, denn Bakterien innerhalb von Biofilmen sind ungefähr 1.000-fach toleranter gegenüber Antibiotika als in Flüssigkultur [6], obwohl diese meistens frei in den Biofilm hineindiffundieren können.

Solche biofilmspezifischen Verhaltensweisen könnten durch konzeptionell unterschiedliche Prozesse entstehen, die unser Verständnis von der Biofilmlebensform stark beeinflussen. Eine Möglichkeit ist eine „additive Multizellularität“, die dadurch entsteht, dass jede einzelne Zelle im Biofilm das gleiche Programm abspult, z. B. bei der Produktion von Matrix. Dadurch können auf makroskopischen Längenskalen neue Eigenschaften der Zellgruppe entstehen, z. B. eine Diffusionslimitierung durch die Matrix oder Veränderungen der mechanischen Materialeigenschaften der Gemeinschaft, die zu den klassischen Faltungen von Biofilmkolonien auf Agaroberflächen führen können. Durch die besonderen mikroskopischen Umweltbedingungen innerhalb eines Biofilms können sich aber auch Genexpressionszustände ausbilden, die nur in Biofilmen existieren. Es entsteht eine „differenzierte Multizellularität“. Diese Möglichkeit ist nicht abwegig, da bereits kleine dreidimensionale Biofilme eine 1.000-fach größere Zelldichte erreichen als Übernachtkulturen. Zellen können innerhalb von Biofilmen somit Umweltbedingungen ausgesetzt sein, die in Flüssigkultur nur schwer zu untersuchen sind.

Differenzierte Multizellularität kann wiederum auf zwei verschiedene Vorgänge zurückzuführen sein [7]: Zum einen könnten Zellen eine Differenzierung durchlaufen, die vererbt wird, ähnlich den Entwicklungsprozessen in Pflanzen oder Tieren. Andererseits könnte sie auch durch die lokale Adaptation der Zellen an jeweilige lokale Umweltbedingungen in verschiedenen Bereichen des Biofilms hervorgerufen werden. Somit wäre das Biofilmwachstum nicht direkt ein Entwicklungsprozess, sondern die Konsequenz des Wachstums einer Zellgruppe.

Langfristig wollen wir die verschiedenen Formen der Multizellularität in Biofilmen unterscheiden, mechanistisch entschlüsseln und die Funktionen von multizellulären Verhaltensweisen von Biofilmen verstehen.

\section{Formen der Multizellularität unterscheiden}

Raumzeitlich variierende Prozesse, wie Biofilmentstehung und -verhalten, erfordern die Erhebung und Analyse von raumzeitlich aufgelösten Daten. Das Mitteln über räumliche Variationen einer Messgrö- ße (z. B. durch räumlich nicht aufgelöste Western Blots) kann die vorhandene phänotypische Heterogenität innerhalb von bakteriellen Gemeinschaften verschleiern. Demzufolge sind, neben Techniken aus der molekularen Mikrobiologie, räumlich-bildgebende Verfahren von zentraler Bedeutung für die Biofilmforschung. Eine natürliche, quantitative und präzise Sprache für die Erfassung, Analyse und Modellierung raumzeitlicher Daten entwickelten die Mathematik und Physik in den letzten Jahrhunderten, auf die man in der Biofilmforschung zurückgreifen kann. Die Biofilmforschung profitiert somit stark von interdisziplinären Ansätzen. 
Um dynamische Prozesse in Biofilmen auf ihre multi- und unizelluläre Ebene herunterzubrechen, ist es notwendig, alle einzelnen Zellen in der Gemeinschaft zu erkennen, diese über die Zeit zu verfolgen sowie Signale und Eigenschaften von einzelnen Zellen zu messen. Hierfür haben wir in den letzten Jahren neue Methoden in der automatisierten Bildanalyse und konfokalen Fluoreszenzmikroskopie entwickelt [8-10]. Diese Methoden ermöglichen eine robuste Erkennung von Zellen in dreidimensionalen Bildern sowie die Quantifizierung von zellulären Eigenschaften und Reportern - sowohl für einzelne Zellen als auch im Kontext der räumlichen Anordnung in Biofilmen [9]. Gekoppelt mit automatisierter Hochdurchsatzmikroskopie, Genetik, Molekularbiologie und mathematischer Analyse ist es nun möglich, im Detail raumzeitliche Phänomene in Biofilmen zu untersuchen und zwischen Einzelzellverhalten und Gruppenverhalten zu unterscheiden (Abb. 1).

\section{Dynamische Prozesse auf mehreren Ebenen in Biofilmen}

Mehrere dynamische Prozesse in Biofilmen haben sowohl eine Komponente auf der Einzelzellebene als auch auf der Multizellebene.

Neben dem Biofilmwachstum ist die selbstinduzierte Biofilmauflösung ein wichtiger Bestandteil des Lebenszyklus von Bakterien in Biofilmen. Zufällig entdeckten wir bei in Strömungskammern gewachsenen Vibrio cholerae-Biofilmen, dass wir die Biofilmauflösung durch das Anhalten der Strömung induzieren können. Die kontrollierte Induktion der Biofilmauflösung ermöglicht eine detaillierte Untersuchung der zugrunde liegenden Mechanismen. $V$. cholerae verbindet ein Signal auf Einzelzellebene (Nährstofflimitierung) mit einem Signal auf Multizellebene (Quorum sensing), um als Kollektiv über die aktive Selbstauflösung des Biofilms zu entscheiden [11].

Ein ähnliches Muster entdeckten wir bei Untersuchungen der Wechselwirkungen von Phagen mit Biofilmen. Escherichia coli-Biofilme sind vor Phagen geschützt, wenn sie eine bestimmte Matrixkomponente ausbilden: Curli-Amyloid-Fibrillen. Diese Fibrillen entstehen hauptsächlich am äußeren Rand des Biofilms, wo sie eine Art Schutzschicht gegen das Eindringen von Phagen in den Biofilm bilden. Curli-Fibrillen können zudem einzelne Zellen tief im Inneren des Biofilms schützen, sofern sich diese komplett mit Curli einhüllen [12]. Diese Amyloid-Fibrillen üben somit eine Funktion sowohl auf der Einzelzellebene als auch auf der Multizellebene aus.
Auch die Stressantwort von Biofilmen auf Antibiotikabehandlung erfolgt auf mehreren Ebenen: Kürzlich entdeckten wir, dass $V$. cholerae-Biofilme als Antwort auf die Behandlung mit Translationsinhibitoren eine dramatische Änderung ihrer Biofilmarchitektur durchlaufen. Diese Änderung konnten wir durch eine Kombination von Experimenten und Simulationen auf eine Mischung aus einer metabolischen Reaktion der einzelnen Zellen und eine Veränderung der Matrixstruktur zurückführen, die auf der vielzelligen Ebene erfolgt.

\section{Zelluläre Wechselwirkungen während der Biofilmentstehung}

In welcher Kombination Einzelzell- und Multizellprozesse das Biofilmwachstum beeinflussen und somit die Biofilmarchitektur und phänotypische Differenzierung der Zellen ermöglichen, gehört zu den großen ungeklärten Fragen in der Biofilmforschung. Vor Kurzem zeigten wir mit einer quantitativen biophysikalischen Studie zur Rolle verschiedener Zell-Zell-Wechselwirkungen bei der V. cholerae-Biofilmentstehung, dass für kleine Biofilme zunächst mechanische Wechselwirkungen dominant sind [9]. Für größere Biofilme ist jedoch noch unklar, welche Wechselwirkungen auf welchen Längenskalen dominieren. Welche Rolle additive und differenzielle Multizellularität in der Entstehung der Biofilmarchitektur einnehmen und inwieweit Gene mit bisher unbekannter Funktion darin involviert sind, muss noch geklärt werden.

Ein Verständnis der verschiedenen Formen der Multizellularität und deren Funktionen innerhalb von Biofilmen könnte unsere Blickweise auf bakterielles Leben und dessen sozialen Kontext grundlegend verändern. Dieser Ansatz bietet somit ein aufregendes Forschungsfeld in der Mikrobiologie.

\section{Danksagung}

Ich bin der deutschen Mikrobiologie-Community und insbesondere der VAAM dankbar für ihre Unterstützung und Offenheit gegenüber meiner interdisziplinären Forschung. Allen ehemaligen und derzeitigen Mitarbeitern meiner Forschungsgruppe bin ich dankerfüllt verbunden für ihren Einsatz und für das starke Gemeinschaftsgefühl, das sie erzeugen. Mein Dank gilt auch der MaxPlanck-Gesellschaft für die große Unterstützung meiner Forschung sowie dem Fachbereich Physik der Universität Marburg, dem Europäischen Forschungsrat, der Deutschen Forschungsgemeinschaft, dem Human Fron- tier Science Program, der Alexander-vonHumboldt-Stiftung sowie der Behrens-WeiseStiftung für die finanzielle Förderung. Antje und Niels bin ich dankbar für ihr Verständnis und ihre Geduld.

Open Access:

This article is distributed under the terms of the Creative Commons Attribution 4.0 International License (http:// creativecommons.org/licenses/by/4.0/), medium or format, as long as you give appropriate credit to the original author(s) and the source, provide a link to the Creative Commons license, and indicate if changes were made. Open access funding provided by Max Planck Society.

\section{Literatur}

[1] Nealson KH, Platt T, Hastings JW (1970) Cellular control of the synthesis and activity of the bacterial luminescent system. J Bacteriol 104:313-322

[2] Nadell CD, Drescher K, Foster KR (2016) Spatial structure, cooperation and competition in biofilms. Nat Rev Microbiol 14:589-600

[3] Parsek MR, Greenberg EP (2005) Sociomicrobiology: the connections between quorum sensing and biofilms. Trends Microbiol 13:27-33

[4] Flemming H-C, Wingender J, Szewzyk U et al. (2016) Biofilms: an emergent form of bacterial life. Nat Rev Microbiol 14:563-575

[5] Flemming H-C, Wuertz S (2019) Bacteria and archaea on Earth and their abundance in biofilms. Nat Rev Microbiol $17: 247-260$

[6] Koo H, Allan RN, Howlin RP et al. (2017) Targeting microbial biofilms: current and prospective therapeutic strategies. Nat Rev Microbiol 15:740-755

[7] Monds RD, O'Toole GA (2009) The developmental model of microbial biofilms: ten years of a paradigm up for review. Trends Microbiol 17:73-87

[8] Drescher K, Dunkel J, Nadell CD et al. (2016) Architectural transitions in Vibrio cholerae biofilms at single-cell resolution. Proc Natl Acad Sci USA 113:E2066-72

[9] Hartmann R, Singh PK, Pearce P et al. (2019) Emergence of three-dimensional order and structure in growing biofilms. Nat Phys 15:251-256

[10] Jeckel H, Jelli E, Hartmann R et al. (2019) Learning the space-time phase diagram of bacterial swarm expansion. Proc Natl Acad Sci USA 116:1489-1494

[11] Singh PK, Bartalomej S, Hartmann R et al. (2017) Vibrio cholerae combines individual and collective sensing to trigger biofilm dispersal. Curr Biol 27:3359-3366

[12] Vidakovic L, Singh PK, Hartmann R et al. (2018) Dynamic biofilm architecture confers individual and collective mechanisms of viral protection. Nat Microbiol 3:26-31

Korrespondenzadresse:

Prof. Dr. Knut Drescher

Max-Planck-Institut für terrestrische Mikrobiologie Zentrum für Synthetische Mikrobiologie

Karl-von-Frisch-Straße 16

D-35043 Marburg

Tel.: 06421-28-21473

k.drescher@mpi-marburg.mpg.de

http://drescherlab.org

\begin{tabular}{|l|l|}
\hline AUTOR & $\begin{array}{l}\text { Knut Drescher } \\
\text { Jahrgang 1983. 2003-2007 } \\
\text { Physikstudium an der Univer- } \\
\text { sity of Oxford, UK. 2007- } \\
2011 \text { biophysikalische Promo- } \\
\text { tion an der University of Cam- } \\
\text { bridge, UK. 2011-2014 Post- } \\
\text { doktorand an der Princeton } \\
\text { University, USA. Seit 2014 } \\
\text { Leiter der Max-Planck-For- }\end{array}$ \\
schungsgruppe „Bakterielle Biofilme“ am Max- \\
Planck-Institut für terrestrische Mikrobiologie, \\
Marburg. Seit 2015 Professor für Biophysik an \\
der Universität Marburg.
\end{tabular}

